Check for updates

Cite this: J. Mater. Chem. A, 2020, 8 , 8234

Received 2nd December 2019

Accepted 1st April 2020

DOI: 10.1039/c9ta13193k

rsc.li/materials-a

\section{Rational design and resolution of the mystery of the structure of Cyclo[18]carbon}

\author{
Mohammad Z. Rahman (iD and Tomas Edvinsson (iD)*
}

$\mathrm{C}_{18}$ is a cyclo[18]carbon that consists of 18 carbon atoms. Beyond the detection of an intermediate gas phase species, the synthesis of a solid-state $\mathrm{C}_{18}$ and elucidation of its bonding structure have remained significant scientific challenges over the past half century. Until recently, this unorthodox carbon allotrope was conceptually feasible, while experimentally elusive in the solid state. Finally, this carbon allotrope has made its long-awaited debut when a collaborative research effort successfully synthesized and revealed the structure of cyclocarbon. The long 50 years of waiting is now over with the unveiling of this new form of chemistry's most celebrated element. This article highlights the discovery of this new allotrope from the carbon family, and discusses its chemical structure, potential applications, and challenges for future research.

\section{Introduction}

Carbon is one of the most abundant elements in the universe. It exists in different forms known as allotropes. Allotropes have different chemical structures that give rise to variations in color, shape, hardness, and electronic properties. Diamond, graphite, graphene, fullerenes, carbon nanotubes, and amorphous carbon are the best known allotropes of carbon. ${ }^{1}$ For example, in diamond, each carbon atom is connected to four other carbon atoms in a network; whereas in graphite (graphene), fullerene, ${ }^{2}$ and carbon nanotubes, ${ }^{3}$ every carbon atom is bonded to three neighboring carbons. In precise terms, graphene is a two-dimensional sheet consisting of a single layer of graphite. ${ }^{4}$ Beyond these well-studied forms of carbon, scientists have also been searching for an allotrope called a cyclo[18]carbon, which consists of a ring of 18 carbon atoms in which each carbon atom connects with only two neighboring atoms..$^{\mathbf{5 , 6}}$

In 1966, Hoffmann proposed that cyclo[n]carbons with $n=$ $[4 q+2]$ carbon atoms, such as $\mathrm{C}_{18}$, may be particularly stable. ${ }^{5}$ Since the late 1980s, the preparation of cyclocarbons has begun from defined macrocyclic dehydroannulenes or expanded radialene precursors. However, these syntheses only succeeded in the gas phase, and bulk quantities were not successfully isolated (progress in carbon allotropes design is shown in Fig. 1). ${ }^{7,8}$ Diederich et al. reported for the first time evidence for the generation of cyclo[18]carbon from a stable organic precursor in the gas phase. ${ }^{6,8}$ However, because of its high reactivity, it could not be isolated and characterized at that time. Its high reactivity causes cyclo[18]carbon to coalesce into other carbon structures; for example, fullerene. ${ }^{9,10}$ Moreover, its

Division of Solid-State Physics, Department of Materials Sciences, Angstrom Laboratory, Uppsala University, Sweden. E-mail: tomas.edvinsson@angstrom.uu.se structure was unknown. Two possibilities were debated regarding whether all the bonds in the ring would be of the same length (double bond) or whether there would be alternating shorter (single bond) and longer bonds (triple bond). ${ }^{6,10}$ These previous studies were tantalizing to scientists hoping to work out the structure of cyclo[18]carbon, with an important description of bond formation.

Recently, a group of researchers from Oxford University and IBM in Zurich reported the successful synthesis, stabilization, and characterization of cyclo[18]carbon, a ring of 18 carbon atoms, on a salt support. ${ }^{11}$ The Oxford team created precursor molecules known as cyclocarbon oxides $\left(\mathrm{C}_{24} \mathrm{O}_{6}\right)$ that provided

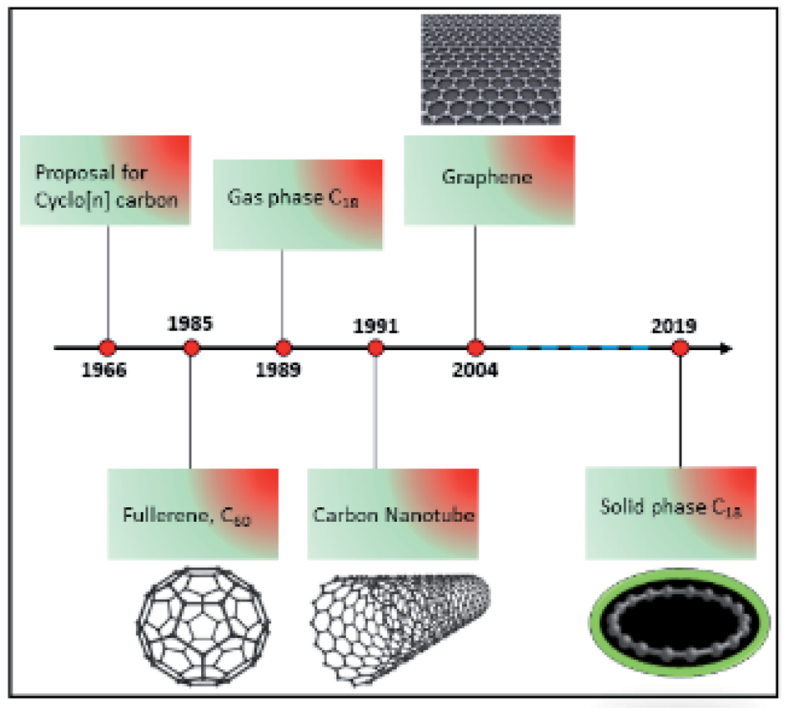

Fig. 1 Chronological progress in the development of different allotropes of carbon, including $\mathrm{C}_{18}$. 
a route to the creation of carbon rings after removal of $\mathrm{CO}$ molecules attached to $\mathrm{C}_{24} \mathrm{O}_{6}$. The IBM researchers used atomic force microscopy (AFM) to knock off the extra molecules by applying voltage pulses to the AFM tip in order to alter the structure of the molecule while it was held on a salt surface at super-cold temperatures. This collaborative effort was finally able to synthesize the $\mathrm{C}_{18}$ ring, and thus find a way to create $\mathrm{C}_{18}$ in the solid state.

\section{Synthesis of $\mathrm{C}_{18}$}

$\mathrm{C}_{18}$ was synthesized based on the imaging of molecules with AFM, and the creation of molecules by atom manipulation on an inert surface at low temperatures $(5 \mathrm{~K}) .{ }^{13}$ Initially, atom manipulation was adopted to synthesize linear segments of twofold coordinated carbons. The chemical reactions were triggered by applying voltage pulses with the tip of the AFM. As such, it was found that such segments could be formed on a copper substrate covered by a very thin layer of table salt. The salt layer ensures a very chemically inert environment so that the reactive molecules do not form covalent bonds to it.

The successful creation of the linear carbon segments inspired to create cyclocarbon on the same surface. To this end, the precursor $\mathrm{C}_{24} \mathrm{O}_{6}$ was synthesized and used for the synthesis of cyclo[18]carbon using atomic manipulation (see the Fig. 2, detail discussed in ref. 11). A total of 90 atomic manipulation events were evaluated.

Extra molecules from $\mathrm{C}_{24} \mathrm{O}_{6}$ were removed using a method called decarbonylation. The most successful conditions for decarbonylation involved large tip-sample distances (small currents; i.e., $I<1 \mathrm{pA}$ ) and bias voltages of about $+3 \mathrm{~V}$. In most cases, two, four, or six $\mathrm{CO}$ molecules were removed using one voltage pulse. Depending on the number of CO molecules removed, the resultant products were either cyclo[18]carbon (if six CO molecules were removed) or an intermediate with an even number of COs still attached. The most abundantly formed intermediates had either one or two intact masking groups (COs). Other molecules less frequently created from $\mathrm{C}_{24} \mathrm{O}_{6}$ by voltage pulses are termed rare intermediates, in which a single CO unit is removed to generate two monoketenes and subsequent rearrangement of the polyynic region to reveal an additional triple bond. The outcomes of all tip-induced decarbonylation attempts starting from either the intact precursor or one of the intermediates are shown in the following reactions, where the yield for the generation of cyclo[18]carbon from the precursor $\mathrm{C}_{24} \mathrm{O}_{6}$ can be seen to be $\sim 13 \%$ :

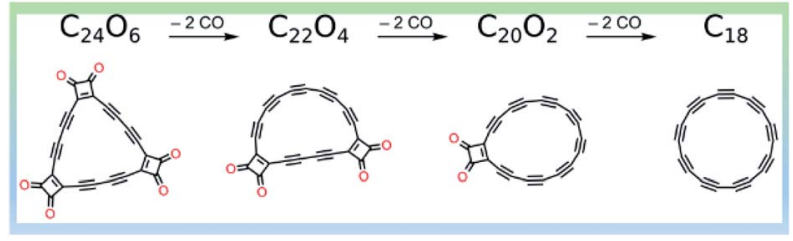

Fig. 2 Synthesis of $\mathrm{C}_{18}$. Reproduced with permission. ${ }^{11}$ Copyright 2019, AAAS.

$$
\begin{gathered}
\mathrm{C}_{24} \mathrm{O}_{6} \rightarrow \mathrm{C}_{18}(4 \% \text { yield }) \\
\mathrm{C}_{24} \mathrm{O}_{6} \rightarrow \mathrm{C}_{20} \mathrm{O}_{2} \rightarrow \mathrm{C}_{18}(3.6 \% \text { yield }) \\
\mathrm{C}_{24} \mathrm{O}_{6} \rightarrow \mathrm{C}_{22} \mathrm{O}_{4} \rightarrow \mathrm{C}_{18}(2.2 \% \text { yield }) \\
\mathrm{C}_{24} \mathrm{O}_{6} \rightarrow \text { rare intermediate } \rightarrow \mathrm{C}_{18}(0.8 \% \text { yield }) \\
\mathrm{C}_{24} \mathrm{O}_{6} \rightarrow \mathrm{C}_{22} \mathrm{O}_{4} \rightarrow \mathrm{C}_{20} \mathrm{O}_{2} \rightarrow \mathrm{C}_{18}(0.7 \% \text { yield }) \\
\mathrm{C}_{24} \mathrm{O}_{6} \rightarrow \mathrm{C}_{20} \mathrm{O}_{2} \rightarrow \text { rare intermediate } \rightarrow \mathrm{C}_{18}(1.1 \% \text { yield }) \\
\mathrm{C}_{24} \mathrm{O}_{6} \rightarrow \mathrm{C}_{22} \mathrm{O}_{4} \rightarrow \text { rare intermediate } \rightarrow \mathrm{C}_{18}(0.2 \% \text { yield }) \\
\mathrm{C}_{24} \mathrm{O}_{6} \rightarrow \text { rare intermediate } \rightarrow \mathrm{C}_{20} \mathrm{O}_{2} \rightarrow \mathrm{C}_{18}(0.1 \% \text { yield }) \\
\text { Total yield of } \mathrm{C}_{18}: 12.7 \%
\end{gathered}
$$

\section{Structure of $\mathrm{C}_{18}$}

The structure of $\mathrm{C}_{18}$ was explored using AFM and density functional theory (DFT) calculations. AFM data and the corresponding simulations for $\mathrm{C}_{24} \mathrm{O}_{6}$ and products created by atom manipulation are shown in Fig. 3. As shown in Fig. $3 \mathrm{~B}$, the $\mathrm{C}_{24} \mathrm{O}_{6}$ molecules appear as triangular objects with dark features at the corners, and two bright protrusions at each side. The dark contrast is characteristic of ketone groups ${ }^{\mathbf{1 2}}$ due to a reduced adsorption height and relatively small electron density in the region imaged above the $\mathrm{O}$ atoms. Two bright features at each side of the molecule can be observed, which are assigned as

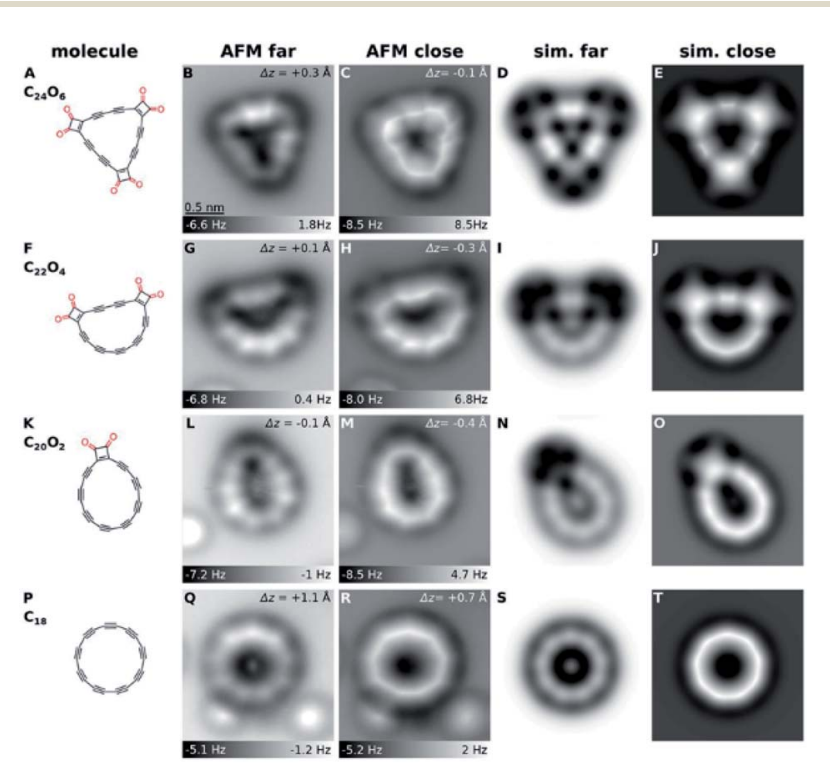

Fig. 3 Structures (1st column) and AFM images ( 2 nd and 3rd columns) of precursor and products generated by tip-induced decarbonylation. ( $A$ to $E$ ) precursor, ( $F$ to $\mathrm{J}$ and $\mathrm{K}$ to $\mathrm{O}$ ) most frequently observed intermediates and ( $P$ to $T$ ) cyclo[18]carbon. The bright features at the lower part in (L), (M), (Q) and (R) correspond to individual $\mathrm{CO}$ molecules. The same scale bar as in (B) applies to all of the experimental and simulated AFM images. Reproduced with permission from. ${ }^{11}$ Copyright 2019, AAAS. 
triple bonds. ${ }^{\mathbf{1 3 , 1 4}}$ When all six CO moieties were removed from $\mathrm{C}_{24} \mathrm{O}_{6}$, this typically resulted in circular $\mathrm{C}_{18}$ molecules with $13 \%$ yield (calculated from the evaluation of 90 atomic manipulation events) (Fig. 3Q and R). When AFM tip heights were moderate, the resulting molecule exhibited a cyclic arrangement of nine bright lobes (Fig. 3Q). For smaller tip heights, the molecule appeared as a nonagon with corners at the positions of the bright lobes (Fig. 3R). The bright lobes in Fig. 3Q and the corresponding corners in Fig. 3R were observed above the triple bonds. The molecule was thus identified as cyclo[18] carbon, $\mathrm{C}_{18}$.

\section{Conclusion and perspective}

The enormous significance of this research is reflected in the fact that it is not every day that a new form of carbon is created in the solid state. Indeed, it took 50 years to bring this highly sought-after form of carbon allotrope to reality. $\mathrm{C}_{18}$ extends the carbon family beyond diamond, graphite, thin sheets called graphene, tiny spheres known as buckyballs, and miniature cylinders called carbon nanotubes. From now on, the textbooks will need to add one more allotrope to the carbon family.

Although previous research had found hints of $\mathrm{C}_{18}$ molecules in a gas phase, it was not possible to image the molecule and confirm its structure. A particular challenge was to gain a clear understanding of whether the bonds between each atom would alternate between longer and shorter lengths, as single and triple bonds, or whether all the bonds would be the same length, as double bonds. Importantly, this issue is now resolved with the conclusion that the carbon atoms in cyclocarbon are held together by alternating single and triple bonds at cold temperatures. To determine if this bonding situation would prevail without the salt support and at higher temperatures requires further research.

The synthesis of $\mathrm{C}_{18}$ and cyclocarbon oxides opens new avenues for the synthesis of other carbon allotropes and carbonrich materials from the coalescence of cyclocarbon molecules; for example, the theoretically predicted $\mathrm{sp}-\mathrm{sp}^{2}$ bonded $3 \mathrm{D}$ microporous carbon. ${ }^{16,17}$

The results suggest the possibility of forming other carbon structures by molecular and atom manipulation to create more sophisticated carbon-rich molecules and new carbon allotropes. Although this has not yet been demonstrated, these rings presumably have potentially exciting electronic properties like graphene. However, our preliminary theoretical results show that, unlike graphene, $\mathrm{C}_{18}$ is not a gapless material. Hybrid DFT calculations on a B3LYP/6-311G(d,p) level shows a static highest occupied molecular orbital (HOMO)-lowest unoccupied molecular orbital (LUMO) gap of $3.1 \mathrm{eV}$, while time-dependent DFT on the same level shows an optical band gap of $2.44 \mathrm{eV}$. Higher order methods and theoretical assessment of the effects from variations in loop length and substitutional closed rings will be interesting in further studies. Therefore, although so far only stabilized at cryogenic conditions, this research potentially holds the promise of applications beyond graphene, and in an optoelectronic range interesting for the development of (cryo) optoelectronics devices. Notably, these custom-made molecular structures might be used as elements for molecular electronics based on single electron transfer, and may eventually be useful in computer technology as an extremely energy-efficient computer logic device. Utilizing resonances or combinations of resonances may additionally open the possibility of multiple state referencing, and thus a possible use in quantum computers at cryogenic temperatures. Before this happens, however, a rational synthesis of stable solid-state $\mathrm{C}_{18}$ must be developed.

Now the challenge of synthesizing an isolated ring is resolved, the next test is to understand its fundamental physicochemical properties, and to determine how to simplify the synthesis and create larger amounts without increasing carbon length, together with routes to make larger-size rings more easily. Here, substrate-stabilized closure of carbyne (linear acetylenic carbon) is an interesting approach. As the optical properties of carbyne change upon bending, ${ }^{15}$ control of the optical properties of cyclocarbon can be expected by varying the radius, and thus the amount of bending.

Previous works on new forms of carbon were received with great excitement and garnered Nobel Prizes. For example, the discovery of buckyballs (fullerene) in the 1980s and the discovery of graphene in 2004 were honored with Noble Prizes. Is the creation of a new solid-state carbon allotrope, cyclocarbon, in the realm of proximity of a Noble Prize? It is too early to speculate its future. At the moment, the foremost challenge is to make this material more stable for further study. Until we understand in detail the fundamental chemical, electronic, and optical properties of cyclocarbon, we cannot say with certainty how widespread its impact will be.

\section{Conflicts of interest}

The authors have no conflicts to declare.

\section{Acknowledgements}

The authors acknowledge generous support from the Swedish Energy Agency (grant no.: 44648-1).

\section{References}

1 V. Georgakilas, J. A. Perman, J. Tucek and R. Zboril, Chem. Rev., 2015, 115, 4744.

2 F. Schedin, E. Lidorikis, A. Lombardo, V. G. Kravets, A. K. Geim, A. N. Grigorenko, K. S. Novoselov and A. C. Ferrari, ACS Nano, 2010, 4, 5617.

3 S. Iijima and T. Ichihashi, Nature, 1993, 363, 603.

4 K. S. Novoselov, A. K. Geim, S. V. Morozov, D. Jiang, Y. Zhang, S. V. Dubonos, I. V. Grigorieva and A. A. Firsov, Science, 2004, 306, 666.

5 R. Hoffmann, Tetrahedron, 1966, 22, 521.

6 F. Diederich and M. Kivala, Adv. Mater., 2010, 22, 803.

7 F. Diederich, Nature, 1994, 369, 199.

8 F. Diederich, Y. Rubin, C. B. Knobler, R. L. Whetten, K. E. Schriver, K. N. Houk and Y. I. Li, Science, 1989, 245, 1088. 
9 G. von Helden, N. G. Gotts and M. T. Bowers, Nature, 1993, 363, 60.

10 S. W. McElvany, M. M. Ross, N. S. Goroff and F. Diederich, Science, 1993, 259, 1594.

11 K. Kaiser, L. M. Scriven, F. Schulz, P. Gawel, L. Gross and H. L. Anderson, Science, 2019, 365, 1299-1301.

12 B. Schuler, W. Liu, A. Tkatchenko, N. Moll, G. Meyer, A. Mistry, D. Fox and L. Gross, Phys. Rev. Lett., 2013, 111, 106103.

13 N. Pavliček, P. Gawel, D. R. Kohn, Z. Majzik, Y. Xiong, G. Meyer, H. L. Anderson and L. Gross, Nat. Chem., 2018, 10, 853 .
14 D. G. de Oteyza, P. Gorman, Y.-C. Chen, S. Wickenburg, A. Riss, D. J. Mowbray, G. Etkin, Z. Pedramrazi, H.-Z. Tsai, A. Rubio, M. F. Crommie and F. R. Fischer, Science, 2013, 340, 1434.

15 A. La Torre, A. Botello-Mendez, W. Baaziz, J.-C. Charlier and F. Banhart, Nat. Commun., 2015, 6, 6636.1.

16 L. Liu, M. Hu, C. Liu, X. Liang, Y. Pan, P. Ying, Z. Zhao, G. Gao, J. He and Y. Tian, Phys. Chem. Chem. Phys., 2018, 20, 15022-15029.

17 L. Liu, M. Hu, Y. Pan, M. Xiong, C. Liu, Y. Zhang, K. Luo, Z. Zhao, G. Gao, D. Yu and J. He, J. Mater. Sci., 2017, 53, 4316-4322. 